\title{
Prevalencia del parasitismo gastrointestinal en bovinos del departamento Cesar, Colombia
}

\author{
Prevalence of gastrointestinal parasitism in bovines of Cesar State, Colombia \\ Juan Carlos Pinilla ${ }^{1,5}$, Paola Flórez ${ }^{2}$, Marly Sierra ${ }^{2}$, Elsa Morales ${ }^{2}$, Raúl Sierra ${ }^{1}$, \\ María Cristina Vásquez ${ }^{2}$, Julio César Tobon ${ }^{3}$, Alfredo Sánchez ${ }^{3}$, Diego Ortiz ${ }^{4}$
}

\section{Resumen}

El objetivo del estudio fue determinar la prevalencia de parásitos gastrointestinales en bovinos del departamento del Cesar, Colombia. Se colectaron 862 muestras fecales en 27 fincas de doble propósito de dos municipios. Los animales fueron estratificados en tres grupos etarios: 0-12, 12-24 y $>24$ meses. Las muestras se procesaron mediante las técnicas coprológicas de McMaster, Dennis y Baermann. Se identificaron los géneros de los parásitos según morfología de sus huevos o larvas infectivas. La prevalencia global de parásitos gastrointestinales fue de $83.2 \%$, siendo los valores más altos para Eimeria sp (77.9\%), Strongyloides sp (10.8\%) y Haemonchus sp (8.5\%). No se encontró asociación estadística por efecto de los municipios, pero se observó por efecto del grupo etario en la prevalencia de Eimeria sp, Strongyloides, Haemonchus y Trichostrongylus $(\mathrm{p}<0.05)$.

Palabras clave: gastrointestinal; helmintos; parásitos; vacunos

\section{AbSTRACT}

The objective of the study was to determine the prevalence of gastrointestinal parasites in bovines from the department of Cesar, Colombia. Faecal samples were collected $(\mathrm{n}=862)$ from 27 dual-purpose cattle farms in two municipalities. The animals were stratified into three age groups: $0-12,12-24$ and $>24$ months. The samples were processed using the coprological techniques of McMaster, Dennis and Baermann. The genera of the parasites were identified according to the morphology of their eggs or

\footnotetext{
${ }^{1}$ Programa de Medicina Veterinaria, ${ }^{2}$ Programa de Bacteriología y Laboratorio Clínico, Universidad de Santander, Lagos de Cacique, Campus Bucaramanga, Colombia

${ }^{3}$ Laboratorio VECOL, Bogotá, Colombia

${ }^{4}$ CORPOICA, Bogotá, Colombia

${ }^{5}$ E-mail: jcpinilla@hotmail.com
}

Recibido: 7 de julio de 2017

Aceptado para publicación: 6 de diciembre de 2017 
infective larvae. The overall prevalence of gastrointestinal parasites was $83.2 \%$, with the highest values for Eimeria sp (77.9\%), Strongyloides sp (10.8\%) and Haemonchus sp $(8.5 \%)$. No statistical association was found due to effect of the municipalities, but it was observed due to the effect of the age group on the prevalence of Eimeria sp, Strongyloides, Haemonchus and Trichostrongylus $(\mathrm{p}<0.05)$.

Key words: gastrointestinal; helminths; parasite; cattle

\section{INTRODUCCIÓN}

Las parasitosis gastrointestinales (PGI) son uno de los problemas sanitarios más importantes en el ganado vacuno a nivel mundial, especialmente las infecciones subclínicas, ya que causan pérdidas económicas por disminución en la producción de leche y carne, e incremento en los costos asociados al tratamiento y control (Mederos y Banchero, 2013). La mayor parte de las PGI en el bovino es ocasionada por protozoarios y helmintos. Dentro de los protozoarios, Eimeria sp es un enterococcidio del phylum Apicomplexa que ocasiona la coccidiosis bovina, infección que se caracteriza clínicamente por diarrea, deshidratación y muerte en animales menores de un año (Díaz de Ramírez et al., 2001) y en animales inmunosuprimidos (Shepelo et al., 2015). Díaz de Ramírez et al. (1998) reportaron $53 \%$ de prevalencia y 813 opg de intensidad de infección por coccidias en bovinos del estado Trujillo, Venezuela, mientras que en el estado de Yucatán (México), Domínguez et al. (1993) y Rodríguez-Vivas et al. (2001) reportaron 86 y $72 \%$ de prevalencia en bovinos, respectivamente.

Los nematodos y cestodos ocasionan problemas de gastroenteritis verminosa caracterizadas clínicamente por diarrea, debilidad, hemorragias y deshidratación (Mawatari et al., 2014). Los nematodos tienen ciclo de vida directo, con una fase en el animal y una externa en los pastos, donde ocurre el desarrollo de huevos hasta el tercer estado larval infectante (Cordero del Campillo et al., 1999). Los géneros de mayor importancia y prevalentes a nivel mundial pertenecen a las familias Trichuridae, Trichostrongylidae, Ancylostomidae, Ascarididae y Strongyloididae (Regassa et al., 2006; Colina et al., 2013). Toxocara vitullorum y Haemonchus contortus son potencialmente graves en animales jóvenes y vacas preñadas (Quiroz et al., 2011). La literatura es abundante en reportes de prevalencia de estas parasitosis (Orjuela et al., 1991; Rodríguez-Vivas et al., 2001; Urdaneta et al., 2011; Colina et al., 2013).

Con relación a Fasciola hepatica, parásito trematodo de distribución mundial que afecta a bovinos, ovinos y ocasionalmente al hombre (Cordero del Campillo et al., 1999), Rojas y Cartín (2016) señalaron 1.8\% de prevalencia en bovinos procedentes de diferentes regiones de Costa Rica, mientras que Soca-Pérez et al. (2016) reportaron prevalencias de $60 \%$ en fincas cubanas, y Recalde-Reyes et al. (2014) reportaron 3.7\% de prevalencia en bovinos Quindio, Colombia.

El Departamento del Cesar, Colombia, es una región agropecuaria y pesquera, siendo el sector ganadero uno de los más importantes en la economía regional, con una participación del 6\% del inventario vacuno a nivel nacional. La región se destaca por su industria láctea; sin embargo, cerca del $60 \%$ de las explotaciones ganaderas están orientadas al doble propósito (carne y leche). Según cifras del Instituto Colombiano Agropecuario (ICA, 2016), el departamento cuenta con una población bovina de 1357512 animales, donde el 10\% se encuentran en los municipios Aguachica y Rio de Oro. En Co- 
lombia es escasa la información epidemiológica acerca del parasitismo gastrointestinal en bovinos, especialmente en la región nororiental del país; por lo tanto, este trabajo tuvo como objetivo determinar la prevalencia parasitaria gastrointestinal en bovinos de doble propósito de los municipios Rio de Oro y Aguachica del departamento de Cesar.

\section{Materiales y Métodos}

\section{Ubicación Geográfica}

El estudio se realizó en los municipios Aguachica $\left(8^{\circ} 182243 \mathrm{~N} 73^{\circ} 362553 \mathrm{O}\right)$ y Rio de Oro ( $8^{\circ} 172303$ N 732 232143 O) del departamento del Cesar. La región presenta una temperatura media anual de $28^{\circ} \mathrm{C}$, una altitud de $800 \mathrm{msnm}$ y una precipitación media anual de $1835 \mathrm{~mm}$, con dos periodos de lluvias bien definidos al año (Gobernación del Cesar, 2016).

\section{Diseño del Muestreo}

Se diseñó un muestreo aleatorio de tipo descriptivo y corte transversal. Entre octubre de 2016 y enero de 2017 (época seca) se visitaron 27 fincas ubicadas en las veredas Márquez del municipio Rio de Oro, y veredas Patiño y Las Piñas del municipio Aguachica. Según los registros de vacunación del ICA (2016), la población censada de los dos municipios fue de 110096 animales, y empleando la fórmula para poblaciones conocidas (Thrusfield, 2005), con una prevalencia esperada de $5 \%$ y un nivel de confianza de $95 \%$ se determinó el requerimiento de 862 muestras fecales. Este número fue distribuido proporcionalmente según la cantidad de animales de las fincas.

Los animales eran de tipo doble propósito, entre 4 meses y 13 años. Se formaron tres grupos etarios: 0-12, 12-24 y >24 meses. La toma de la muestra $(10 \mathrm{~g})$ se realizó por vía rectal en horas de la mañana, utilizando bolsas de polietileno. En algunos ca- sos se tomaron muestras de deposiciones recientes. Las muestras se almacenaron en refrigeración y fueron enviadas al Laboratorio de Microbiología de la Facultad de Ciencias de la Salud de la Universidad de Santander, en Bucaramanga, para su procesamiento dentro de los tres días de tomadas las muestras.

\section{Procedimientos Parasitológicos}

Las muestras se procesaron mediante tres técnicas copro-parasitológicas:

- Técnica de McMaster para detección de ooquistes de coccidias (opg) y huevos de nematodos (hpg), empleando una solución de flotación de azúcar-sal ( 1 L de solución saturada de $\mathrm{NaCl}$ más $500 \mathrm{~g}$ de azúcar) (Henriksen y Christensen, 1992; Sandoval et al., 2011). La intensidad de infección se determinó multiplicando por 50 la cantidad de huevos y ooquistes contabilizados en los dos retículos de la cámara, que es el factor estandarizado para esta técnica (Sandoval et al., 2011).

- Coprocultivo mediante la técnica de Baermann a todas las muestras positivas a la técnica de McMaster para la recuperación y determinación de larvas infectivas $\left(\mathrm{L}_{3}\right)$ de nematodos gastrointestinales (Fiel et al., 2011).

- Técnica de Denis modificada (Correa et al., 2016) para la determinación de huevos pesados de $F$. hepatica y Paramphistomum sp.

Los géneros de nematodos y trematodos fueron identificados por la morfología y tamaño de sus huevos y larvas (Fiel et al., 2011), empleando un microscopio óptico de luz con magnificación de 10 y 40x.

\section{Análisis Estadístico}

La prevalencia de las parasitosis se determinó dividiendo el número de animales positivos entre el total de animales de la población muestreada. Los resultados obtenidos se analizaron mediante estadísticos des- 
Cuadro 1. Prevalencia de parasitismo gastrointestinal en bovinos de doble propósito de los municipios Rio de Oro y Aguachica, departamento del Cesar, Colombia

\begin{tabular}{llccc}
\hline \multirow{2}{*}{ Municipio } & \multirow{2}{*}{ Vereda } & \multicolumn{2}{c}{ Muestras $(\mathrm{n})$} & Prevalencia \\
\cline { 3 - 4 } & & Total & Positivos & $(\%)$ \\
\hline Aguachica & Patiño y Las Piñas & 604 & 511 & 84.6 \\
Rio de Oro & Márquez & 258 & 206 & 79.8 \\
\hline Total & & 862 & 717 & 83.2 \\
\hline
\end{tabular}

$X^{2}=2.92 ;$ sin significancia estadística $(p>0.05)$

Cuadro 2. Prevalencia e intensidad de infección de géneros parasitarios diagnosticados en 862 bovinos de los municipios Rio de Oro y Aguachica, departamento del Cesar, Colombia

\begin{tabular}{lccc}
\hline Géneros & $\begin{array}{c}\text { Positivos } \\
(\mathrm{n})\end{array}$ & $\begin{array}{c}\text { Prevalencia } \\
(\%)\end{array}$ & $\begin{array}{c}\text { Intensidad de } \\
\text { infección } \\
\text { (opg-hpg) }\end{array}$ \\
\hline Eimeria & 672 & 77.9 & 530.2 \\
Strongyloides & 93 & 10.8 & - \\
Haemonchus & 73 & 8.5 & 145.6 \\
Fasciola hepatica & 30 & 3.4 & - \\
Trichostrongylus & 26 & 3.1 & 300 \\
Ostertagia & 20 & 2.3 & 226.1 \\
Moniezia & 10 & 1.2 & - \\
Toxocara & 9 & 1.1 & 76.9 \\
Nematodirus & 7 & 0.8 & 87.5 \\
Paramphistomum & 6 & 0.7 & 88.5 \\
Capillaria & 3 & 0.3 & 83.3 \\
Trichuris & 3 & 0.3 & 417 \\
Cooperia & 2 & 0.2 & 75 \\
\hline
\end{tabular}

${ }^{1}$ Strongyloides sp y $F$. hepatica no fueron incluidos en el análisis opg: ooquistes por gramo de heces; hpg: huevos por gramo de heces

criptivos y test de Chi-cuadrado para determinar asociaciones estadísticas. Para los cálculos se utilizó el programa estadístico Statistix v. 8.0.

\section{Resultados}

La prevalencia global del parasitismo gastrointestinal en bovinos de los municipios Aguachica y Rio de Oro fue de $83.2 \%$ (Cua- dro 1). No se encontró asociación estadística $\left(X^{2}=2.92\right)$ entre los valores de prevalencia con respecto a los dos municipios, indicando que la prevalencia de parasitismo gastrointestinal se encuentra presente en proporciones similares en los dos municipios del departamento del Cesar.

En el Cuadro 2 se muestran los trece géneros parasitarios encontrados en el estudio, de los cuales los más prevalentes fueron 
Cuadro 3. Prevalencia de parasitismo e intensidad de la infección por Eimeria sp de acuerdo al grupo etario en bovinos de los municipios Rio de Oro y Aguachica, departamento del Cesar (Colombia)

\begin{tabular}{lccccc}
\hline \multirow{2}{*}{ Grupo etario } & Bovinos & Prevalencia de & \multicolumn{3}{c}{ Valores de opg $^{2}$} \\
\cline { 4 - 6 } & $(\mathrm{n})$ & PGI $^{1}(\%)$ & Media \pm d.e. & Mínimo & Máximo \\
\hline $0-12$ meses & 183 & $65.3^{\mathrm{a}}$ & $440.9 \pm 268.3^{\mathrm{a}}$ & 100 & 1100 \\
$12-24$ meses & 188 & $73.8^{\mathrm{b}}$ & $656.8 \pm 397.6^{\mathrm{a}}$ & 100 & 1900 \\
$>24$ meses & 491 & $79.8^{\mathrm{b}}$ & $527.1 \pm 435.7^{\mathrm{a}}$ & 50 & 4000 \\
\hline Total & 862 & 77.9 & & & \\
\hline
\end{tabular}

${ }^{1}$ Parásitos gastrointestinales

2 opg: ooquistes por gramo de heces

Medias con superíndice igual no difieren significativamente $(p>0.05)$

Cuadro 4. Comparación entre la prevalencia (\%) de parasitismo por trematodos y cestodos de acuerdo al grupo etario en bovinos de los municipios Rio de Oro y Aguachica, Cesar (Colombia)

\begin{tabular}{lcccc}
\hline Grupo etario & Bovinos (n) & $\begin{array}{c}\text { Fasciola } \\
\text { hepatica }\end{array}$ & $\begin{array}{c}\text { Paramphistomun } \\
\mathrm{sp}\end{array}$ & Moniezia $\mathrm{sp}$ \\
\hline $0-12$ meses & 83 & 2.2 & 0 & 2.2 \\
$12-24$ meses & 88 & 5.6 & 1.1 & 1.1 \\
$>24$ meses & 691 & 3.4 & 0.7 & 1.1 \\
\hline Total & 862 & 3.4 & 0.7 & 1.2 \\
\hline & & $\mathrm{X}^{2}=5.9 ; \mathrm{p}=0.11$ & $\mathrm{X}^{2}=0.84 ; \mathrm{p}=0.84$ & $\mathrm{X}^{2}=0.89 ; \mathrm{p}=0.82$
\end{tabular}

Cuadro 5. Prevalencia de parasitismo por nematodos de acuerdo al grupo etario en bovinos de los municipios Rio de Oro y Aguachica, departamento del Cesar, Colombia

\begin{tabular}{|c|c|c|c|c|c|c|c|c|}
\hline Grupo etario & Total & 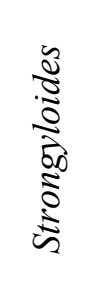 & 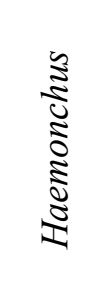 & 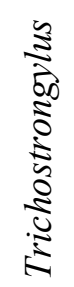 & 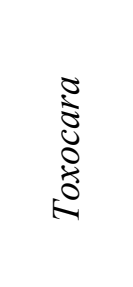 & 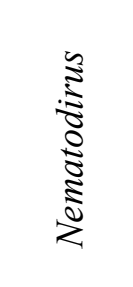 & 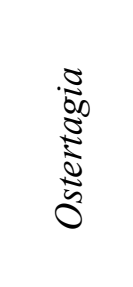 & $\begin{array}{l}-10 \\
0 \\
0 \\
0\end{array}$ \\
\hline $0-12$ meses & 183 & 20.4 & 19.2 & 9.6 & 3.4 & 0 & 3.6 & 4.4 \\
\hline 12-24 meses & 188 & 5.7 & 6.8 & 0 & 0 & 2.3 & 2.3 & 1.1 \\
\hline$>24$ meses & 491 & 10.3 & 7.4 & 4.2 & 0.9 & 0.7 & 2.2 & 0.7 \\
\hline \multirow[t]{2}{*}{ Total } & 862 & 10.8 & 8.5 & 3.0 & 1.0 & 0.8 & 2.3 & 0.9 \\
\hline & & \multicolumn{3}{|c|}{$\mathrm{p}=0.001$} & $\mathrm{p}=0.11$ & $\mathrm{p}=0.37$ & $\mathrm{p}=0.67$ & $\mathrm{p}=0.08$ \\
\hline
\end{tabular}

${ }^{1}$ Capillaria sp, Trichuris sp y Cooperia $s p$ 
Eimeria sp (77.9\%), Strongyloides sp $(10.8 \%)$ y Haemonchus sp (8.5\%). Con respecto a la intensidad de infección, Trichuris sp mostró la mayor intensidad de infección (417 hpg), seguido de Trichostrongylus sp (300 hpg). Es importante indicar que no se contabilizaron los huevos de Strongyloides sp, F. hepatica y Monieza sp. En el 76.1\% de las muestras examinadas se encontró evidencia de parásitos un solo género, en el $20.9 \%$ de dos géneros y en el $3.0 \%$ de más de dos géneros $\left(X^{2}=57.39 ; p<0.05\right)$.

En el parasitismo por Eimeria sp se observó asociación estadística $(\mathrm{p}<0.05)$ con respecto al grupo etario (Cuadro 3), donde los animales de 0 a 12 meses mostraron la menor prevalencia $(65.3 \%)$ y aquellos entre 12 y 24 y $>24$ meses presentaron prevalencias de 73.8 y $79.8 \%$, respectivamente. Por otro lado, no se encontró asociación estadística entre la intensidad de la infección con respecto a la edad de los animales (Cuadro 3). Tampoco se encontró asociación estadística entre $F$. hepatica, Paramphistomun sp y Moniezia sp por grupos etarios (Cuadro 4).

En el Cuadro 5 se observan los valores de prevalencia de los nematodos observados durante el estudio. El género Strongyloides presentó mayor prevalencia $(10.8 \%)$, seguido de Haemonchus sp (8.5\%). Se encontró asociación estadística $(\mathrm{p}<0.01)$ entre las prevalencias de Strongyloides sp, Haemonchus sp y Trichostrongylus sp con respecto al grupo etario. Por otra parte, no se encontró asociación estadística entre los grados de positividad de Ostertagia sp, Toxocara sp, Nematodirus sp y otros parásitos (Capillaria sp, Trichuris sp y Cooperia sp) con respecto a la edad de los animales. La mayor prevalencia de Strongyloides sp, Haemonchus sp y Trichostrongylus sp se observó en animales de 0 a 12 meses de edad.

\section{Discusión}

La presencia de parásitos gastrointestinales en ganado vacuno, así como el grado- de daño causado en el hospedero dependen en gran medida de factores predisponentes, tales como temperatura y humedad. Así, parásitos de los géneros Ostertagia y Dictyocalus prevalecen en climas fríos, mientras que Haemonchus y Cooperia se relacionan con climas tropicales (Quiroz et al., 2011). La alta prevalencia de parásitos gastrointestinales en este estudio (83.2\%) coincide con lo señalado por Urdaneta et al. (2011) y Colina et al. (2013), quienes reportaron altas prevalencias en ganado vacuno en diferentes regiones con condiciones climáticas semejantes a las del presente estudio. Las características del medio ambiente, edad y estado fisiológico de los animales, tipo de pasturas y prácticas agrícolas son factores predisponentes para una alta prevalencia (Colina et al., 2013).

La mayor prevalencia de protozoarios del género Eimeria (77.9\%) y de parásitos del sub-orden Strongylida (16.6\%) y de Strongyloides sp (10.8\%) coincide con lo señalado por Domínguez et al. (1993) en bovinos de Yucatán, México, y con las prevalencias de 53 y $71.5 \%$ de coccidias reportadas por Díaz de Ramírez et al. (1998) y Rodríguez-Vivas et al. (2001), respectivamente; sin embargo, Orjuela et al. (1991) y Rodríguez-Vivas et al. (2001) señalaron mayores valores de prevalencia de parásitos del orden sub-Strongylida en el Norte de Colombia y México, respectivamente.

Aunque la media de intensidad de infección por Eimeria sp encontrada en este estudio (530.2 opg) se considera ligera o baja (Quiroz et al., 2011), se infiere que la alta prevalencia señalada puede estar ocasionada por inmunosupresión en animales debido a factores tales como el estrés por sobrepoblación, transporte y traslados del rebaño (Shepelo et al., 2015). Igualmente, la infección por coccidias en bovinos podría deberse a su capacidad de adaptación a las diferentes condiciones climáticas, como también a un mal manejo de pastoreo y a la contaminación del agua por los animales adultos parasitados (Tamasaukas et al., 2010). 
La mayor prevalencia de Eimeria sp en animales adultos (12-24 y $>24$ meses) encontrada en el presente estudio (73.8 y $79.8 \%$, respectivamente) contrasta con los datos de Díaz de Ramírez et al. (1998) y Tomczuk et al. (2015), quienes reportaron mayor excreción de ooquistes en animales menores de 12 meses, ya que la mayoría de especies de Eimeria de bovinos tienen periodos prepatentes de dos y tres semanas, de modo que los becerros pueden ingerir una cantidad suficiente de ooquistes esporulados para establecer una infección patente en el rebaño (Díaz de Ramírez et al., 1998). En este estudio, los bovinos adultos se podrían comportar como portadores asintomáticos y potenciales fuentes de infección para los becerros, ya que se infectan al ingerir ooquistes esporulados con el alimento o agua o por lamer el pelo de animales con heces contaminadas (Quiroz et al., 2001).

Es posible que la baja intensidad de infección encontrada se encuentre asociada al periodo seco del año, que es cuando se tomaron las muestras. Rodríguez-Vivas et al. (1996) encontraron en el trópico mexicano que uno de los factores asociados a la mayor excreción de ooquistes de Eimeria en el ganado bovino era la época de lluvias.

Según Soulsby (1985), la presencia de infecciones parasitarias mixtas en ganado vacuno es más común que las infecciones monoespecíficas, y se registran en casos de brotes epidémicos, o por efecto del clima de una región determinada. En forma similar, Soca et al. (2005) señalaron $54.4 \%$ de poli parasitismo por nematodos gastrointestinales en un mismo hospedero. Sin embargo, en este estudio, el $76.1 \%$ de los animales estuvieron afectados por parásitos de un solo género. Es probable que debido a la gran variedad de especies parasitarias encontradas en diferentes estudios se pueda esperar la presentación de diversas combinaciones parasitarias; no obstante, animales bajo control parasitario intensivo pueden mostrar mayor frecuencia de infecciones monoespecíficas (Soulsby, 1987).
Con respecto al grupo de nematodos, larvas de Strongyloides sp fueron recuperadas en todos los grupos etarios; pero con mayor frecuencia en animales menores de un año. Estos resultados coinciden con lo señalado por Domínguez et al. (1993), Van Aken et al. (2000) y Urdaneta et al. (2011), quienes demostraron disminución de la prevalencia con el avance de la edad de los animales. Esto puede deberse a que entre las formas de transmisión de Strongyloides sp se encuentran la percutánea y la maternal (lactogénica), de allí que con el hacinamiento y las malas condiciones higiénicas y sanitarias de las fincas se favorece un mayor riesgo de infección en las becerreras. Además, se ha demostrado que la respuesta inmune contra nematodos gastrointestinales madura con la edad del animal (Quiroz et al., 2011).

Dentro del sub-orden Strongylida, el género Haemonchus resultó ser el más prevalente $(8.5 \%)$, tal y como es señalado por Domínguez et al. (1993) en bovinos del estado de Yucatán, México. En forma similar a los Strongyloides, la mayor prevalencia se presenta en animales menores de un año (Urdaneta et al., 2011), posiblemente debido a la mayor fertilidad y periodo prepatente más corto de Haemonchus (Angulo-Cubillán et al., 2007). Es importante mencionar que el potencial de la acción hematófaga de estos parásitos está directamente proporcional a la intensidad de infección presente en el rebaño; es decir, mayor carga parasitaria, mayor patogenicidad (Cordero del Campillo et al., 1999). Los niveles de prevalencia de otros nematodos fueron bajos; sin embargo, se reconoce que las infecciones por Toxocara sp son potencialmente fatales, sobre todo en animales jóvenes (Quiroz et al., 2011).

La baja frecuencia de Moniezia sp y sin deferencias entre grupos etarios fue similar a lo señalado por Orjuela et al. (1991) y Rodríguez-Vivas et al. (2001). La monieziosis se presenta en bovinos a pastoreo, donde existen animales infectados que contaminan los pastos con huevos del cestodo y donde ocurre la presencia de ácaros oribátidos (hos- 
pederos intermediarios), de tal forma que se pueda mantener el ciclo de vida del parásito (Quiroz et al., 2011).

Las prevalencias de $3.4 \%$ de $F$. hepatica coincide con los datos de RecaldeReyes et al. (2014) y Rojas y Cartín (2016) en bovinos del departamento del Quindio, Colombia, y en diferentes regiones de Costa Rica, respectivamente. Sin embargo, fueron bastante inferiores al 58.3-62.5\% de prevalencia en fincas de Cuba (Soca-Pérez et al., 2016), así como al $25 \%$ de prevalencia en Nariño y más del $80 \%$ en algunas zonas de Cundinamarca y Nariño (Recalde-Reyes et al., 2014). Aunque no existen antecedentes de estudios sobre prevalencia y decomisos de hígado de animales provenientes de la zona en estudio, la baja prevalencia de $F$. hepatica encontrada (3.4\%) podría deberse a la poca interacción entre los animales susceptibles y los hospederos intermediarios (Rojas y Cartín, 2016).

En Colombia se han registrado paramfistómidos de los géneros Paramphistomum y Cotylophorum en bovinos de la Costa Caribe y Llanos Orientales (Parra et al., 1982) y en fincas de Río Negro, Antioquia (Alarcón y Velásquez, 2009). La semejanza en las características morfológicas de las especies de paramfistómidos, así como la falta de experiencia en estas especies se convierte en un obstáculo para el desarrollo de estudios epidemiológicos y de prevalencia, por lo que se propone incluir el uso de secuencias genómicas (López y Velásquez, 2009). Se infiere que la baja prevalencia de este trematodo en las localidades de Aguachica y Rio de Oro está determinada por la baja ocurrencia de los moluscos hospedadores intermediarios (caracoles del género Lymnaea).

\section{Agradecimientos}

Los autores agradecen al personal del Laboratorio de Investigación de los programas Bacteriología y Medicina Veterinaria de la Universidad de Santander por la colaboración prestada en el desarrollo de este pro- yecto. Igualmente, se agradece al personal directivo y técnico de VECOL por la colaboración y financiamiento del proyecto, así como a los integrantes del semillero en Salud Pública y Ambiental por su arduo trabajo en esta investigación.

\section{Conclusiones}

- Los bovinos de los municipios Aguachica y Rio de Oro, Cesar, Colombia, se encuentran infectados por una gran variedad de parásitos gastrointestinales, principalmente de los géneros Eimeria, Strongyloides y Haemonchus.

- La alta prevalencia de Eimeria sp en animales adultos indica que este grupo puede comportarse como potencial fuente de infección para los terneros.

\section{Literatura Citada}

1. Alarcón P, Velásquez L. 2009. Descripción morfológica de Cotylophoron cotylophorum (Digenea: Paramphistomidae) hallado en bovinos de Rio negro, Antioquia, Colombia. Rev Colom Cienc Pecua 22: 168-177.

2. Angulo-Cubillán F, García L, Cuquerella M, De La Fuente C, Alunde J. 2007. Haemonchus contortus - sheep relationship: a review. Rev Cien FCV-LUZ 17: 577-587.

3. Colina J, Mendoza G, Jara C. 2013. Prevalencia e intensidad del parasitismo gastrointestinal por nematodos en bovinos, Bos taurus, del Distrito Pacanga (La Libertad, Perú). Rebiol 33(2): 76-83.

4. Cordero del Campillo M, Rojo F, Martínez Av Sánchez C, Hernández $S$, Navarrete I, Díez P, et al. 1999. Parasitología veterinaria. Madrid, España: Ed McGraw-Hill Interamericana. 935 p.

5. Correa S, Martínez Y, López J, Velásquez L. 2016. Evaluación de la técnica modificada de Dennis para el diagnóstico de fasciolosis bovina. Biomed 
36: 64-68. doi: 10.7705/biomedica.v36i 2.2875

6. Díaz de Ramírez A, Hernández A, García A, Ramírez-Iglesia LN. 2001. Excretion of oocysts of Eimeria spp during the first three months of lice in calves from dairy farms in western Venezuela. Rev Cien FCV-LUZ 11: 207-212.

7. Días de Ramírez A, Justo JA, González M, Piña EF, Ramírez LI. 1998. Prevalencia de coccidios en bovinos de Los Llanos de Monay, estado Trujillo, Venezuela. Rev Cien FCV-LUZ 8:346-353.

8. Domínguez JL, Rodríguez RI, Honhold N. 1993. Epizootiología de los parásitos gastrointestinales en bovinos del estado de Yucatán. Vet Méx 24: 189-193.

9. Fiel C, Steffan P, Ferreyra P. 2011. Diagnóstico más frecuente de las parasitosis de los rumiantes: técnicas de laboratorio e interpretación de resultados. Buenos Aires. Argentina: Pfizer. $131 \mathrm{p}$.

10. Gobernación del Cesar. 2016. Municipios del departamento del Cesar. [Internet]. Disponible en: http:// cesar.gov.co/d/index.php/es/ mainmeneldpto/mendepmun

11. Henriksen S, Christensen J. 1992. Demonstration of Isospora suis oocysts in faecal samples. Vet Rec 131: 443-444.

12. [ICA] Instituto Colombiano Agropecuario. 2016. Censo pecuario nacional -2016 . Censo bovino en Colombia. Tabla de población bovina por municipio y por departamento. [Internet]. Disponible en: https://www.ica.gov.co/getdoc/ 8232c0e5-be97-42bd-b07b-9cdbfb07 fcac/Censos-2012.aspx

13. López J, Velásquez L. 2009. Cotylophoron panamensis (Digenea: Paramphistomidae) en bovinos del Meta y del Guaviare, Colombia. Acta Biol Colom 17: 421-430.

14. Mawatari T, Hirano K, Ikeda H, Tsunemitsu H, Suzuki T. 2014. Surveillance of diarrhea-causing pathogens in dairy and beef cows in Yamagata Prefecture, Japan from 2002 to 2011.
Microbiol Immunol 58: 530-535. doi: 10.1111/1348-0421.12174

15. Mederos A, Banchero G. 2013. Parasitosis gastrointestinales en ovinos y bovinos: situación actual y avances de la investigación. Rev INIA 34: 10-15.

16. Orjuela J, Navarrete M, Betancourt A, Roqueme L, Cortez E, Morrison R. 1991. Salud y productividad en bovinos de la costa norte de Colombia. World Animal Rev 69(4): 7-14.

17. Parra D, Gallego M, Griffiths I. 1982. Prevalencia de la paramfistomiasis bovina en hatos lecheros de Colombia. Rev Col Cienc Pec 982: 33-34.

18. Quiroz H, Figueroa J, Ibarra F, López M. 2011. Epidemiología de enfermedades parasitarias en animales domésticos. México: UNAM. 655 p.

19. Recalde-Reyes D, Padilla L, Giraldo M, Toro L, Gonzalez M, Castaño J. 2014. Prevalencia de Fasciola hepatica en humanos y bovinos en el departamento del Quindío-Colombia 2012-2013. Infectio 18: 153-157. doi: 10.1016/j.infect.2014.09.001

20. Regassa F, Sori T, Dhuguma R, Kiros Y. 2006. Epidemiology of gastrointestinal parasites of ruminants in Western Oromia, Ethiopia. Intern J Appl Res Vet Med 4: 51-57.

21. Rodríguez-Vivas RI, DomínguezAlpizar JL, Torres-Acosta JF. 1996. Epidemiological factors associated to bovine coccidiosis in calves (Bos indicus) in a sub-humid tropical climate. Rev Biomed 7: 211-218.

22. Rodríguez-Vivas RI, Ligia A, Cob-Galera LA, Domínguez-Alpizar $J L$. 2001. Frecuencia de parásitos gastrointestinales en animales domésticos diagnosticados en Yucatán, México. Rev Biomed 12: 19-25.

23. Rojas D, Cartín J. 2016. Prevalencia de Fasciola hepatica y pérdidas económicas asociadas al decomiso de hígados en tres mataderos de clase A de Costa Rica. Agronomía Costarricense 40: 53-62. 
24. Sandoval E, Morales G, Ybarra N, Barrios M, Borges J. 2011. Comparación entre dos modelos diferentes de cámaras de McMaster empleadas para el conteo coproscópico en el diagnóstico de infecciones por nematodos gastroentéricos en rumiantes. Zootecnia Trop 29: 495-501.

25. Shepelo P, Gitau G, Matiku C, Vanleeuwen J, Richards S, Wichtel J, Uehlinger F, et al. 2015. Prevalence of Cryptosporidia, Eimeria, Giardia, and Strongyloides in pre-weaned calves on smallholder dairy farms in Mukurwe-ini district, Kenya. Vet World 8: 1118-1125. doi: 10.14202/vetworld.2015.1118-1125

26. Soca M, Roque E, Soca M. 2005. Epizootiología de los nemátodos gastrointestinales de los bovinos jóvenes. Pastos y Forrajes 28: 175-185.

27. Soca-Pérez M, Giupponi-Cardoso P, López-Vigoa O, Sanavria A, SánchezSantana T, Labrada-Vázquez A. 2016. Prevalencia de Fasciola hepatica en vacas en pastoreo durante el periodo poco lluvioso. Pastos y Forrajes 39: 281-285.

28. Soulsby EJ. 1987. Parasitología y enfermedades parasitarias de los animales domésticos. México: Ed Interamericana. $823 \mathrm{p}$.
29. Tamasaukas R, Agudo L, Vintimilla M. 2010. Patología de la coccidiosis bovina en Venezuela: una revisión. REDVET 11(7). [Internet]. Disponible en: http://www.veterinaria.org/revistas/ redvet/n070710/071006.pdf

30. Tomczuk K, Grzybek M, Szczepaniak K, Studziñska M, Demkowska M, Roczen M, Klockiewicz M. 2015. Analysis of intrinsic and extrinsic factors influencing the dynamics of bovine Eimeria spp from central-eastern Poland. Vet Parasitol 214: 22-28. doi: 10.1016/j.vetpar.2015.09.027

31. Thrusfield M. 2005. Veterinary epidemiology. Oxford, England: Ed Blackwell Science. 592 p.

32. Urdaneta M, Urdaneta A, Parra A, Chacín E, Ramírez R, Angulo F. 2011. Prevalencia y grado de infección de helmintos gastrointestinales en rebaños bovinos doble propósito del municipio Miranda del estado Zulia, Venezuela. Rev Cien FCV-LUZ 2: 184-193.

33. Van Aken D, Dargantes A, Valdez L, Flores A, Dorny P, Vercruysse J. 2000. Comparative study of strongyle infections of cattle and buffaloes in Mindanao, the Phillipines. Vet Parasitol 89: 133-137. 